\title{
Large-Scale Beard Extraction Enhances the Cosmetic Results of Scalp Hair Restoration in Advanced Androgenetic Alopecia in East Asian Men: A Retrospective Study
}

\author{
De-Cong Zhu • Ye He - Zhe-Xiang Fan · Jin Wang - Qian Qu • \\ Zhi-Qi Hu • Yong Miao (D)
}

Received: October 5, 2019 / Published online: November 29, 2019

(C) The Author(s) 2019

\begin{abstract}
Introduction: Beard hair serves as an important additional donor supply to support hair transplantation in hirsute patients with extensive alopecia and lacking sufficient occipital hair. However, the efficacy and safety of large-scale beard hair extraction have not been studied extensively in the East Asian population.

Methods: Data obtained from hirsute patients with extensive alopecia who underwent hair transplantation between March 2017 and December 2018 at Nanfang Hospital were analyzed. Occipital and beard hair were evaluated separately during the pre-, intra-, and post-operative periods. Individual beard hair follicular units (FUs) were harvested under tumescence using a hollow punch with an outer diameter of 0.8-0.9 mm. Follow-up examinations were scheduled at 3-5 days, 1 month, and 9 months postoperatively to check for complications, determine the survival rate of mixed,
\end{abstract}

Enhanced Digital Features To view enhanced digital features for this article go to https://doi.org/10.6084/ m9.figshare.10265996.

D.-C. Zhu · Y. He · Z.-X. Fan ·J. Wang · Q. Qu ·

Z.-Q. Hu ( $\square)$. Y. Miao ( $\square)$

Department of Plastic and Aesthetic Surgery,

Nanfang Hospital of Southern Medical University,

Guangzhou, Guangdong 510515, China

e-mail: huzhiqidr@163.comY. Miao

e-mail: doctormiao0371@163.com transplanted FU grafts, and assess patient satisfaction. Data were collected and analyzed statistically.

Results: A total of 36 hirsute, male patients with advanced androgenetic alopecia (AGA) (Norwood-Hamilton V-VI) were included in this study. The density of the occipital and beard areas was $78.6 \pm 4.6$ and $48.4 \pm 9.3 \mathrm{FU}$, respectively. It took $3.1 \pm 0.9 \mathrm{~h}$ to harvest $3135 \pm 863$ FUs from the occipital area and $2.1 \pm 0.6 \mathrm{~h}$ to harvest $2352 \pm 599 \mathrm{FUs}$ from the beard area. The transection rate for occipital FUs and beard FUs was $3.7 \pm 0.4$ and $3.9 \pm 0.2 \%$, respectively. Completion of the operation took approximately $10.0 \pm 0.9 \mathrm{~h}$, and no serious complications were reported 5 days after the procedure. An FU survival rate of $95.7 \pm 1.6 \%$ was observed at 9 months after transplantation, with no visible hypopigmented scars observed in the bare areas. All patients were satisfied with the resulting cosmetic appearance.

Conclusion: Large-scale beard extraction, when combined with occipital hair extraction, is a safe and effective treatment to enhance the cosmetic appearance of East Asian men with advanced AGA.

Keywords: East Asian; Ethnicity; Follicular unit; Hairline area; Hair extraction; Hair transplantation; Hypopigmentation; Largescale beard 


\section{Key Summary Points}

\section{Why carry out this study?}

Androgenetic alopecia (AGA) is the most common type of hair loss disorder in men, affecting $80 \%$ of men by the age of 70 years.

In this work, we asked whether largescale beard hair extraction, combined with occipital hair grafts, could serve as a safe and efficient treatment in advanced AGA patients of East Asian background to enhance the cosmetic result.

\section{What was learned from the study?}

We concluded that large-scale beard hair extraction is a safe and efficient procedure in East Asian people with advanced AGA, leaving no visible hypopigmented scar in the beard area and supplying $2352 \pm 599$ beard follicular units (FU).

When taking beard as the supplementary grafts for advanced AGA, ethnicity ought to be taken into consideration to avoid potential hypopigmentation in the exposed beard area.

If possible, beard FUs, in combination with occipital FUs, could considerably enhance the cosmetic result.

\section{INTRODUCTION}

Autologous hair transplantation has been the safest and most effective treatment for the majority of medical conditions that cause hair loss $[1,2]$. In traditional transplantation surgery, the occipital area serves as a safe donor zone, and the average survival rate of transplanted hair follicle units (FUs) approaches 95\% $[3,4]$. However, for patients with severe alopecia, an inadequate number of transplantable FUs from the occipital area could compromise the cosmetic results. For such

patients, the FU extraction technique extends possible donor sites from the occipital area to regions of body hair, thereforeby increasing the number of FUs that are available for grafting [5-10]. Beard hair, in particular, has been utilized more than hair from other parts of the body, including armpit hair, limb hair, chest hair, and pubic hair [11].

In the earliest hair transplantation procedures involving beard hair, single follicular micrografts of beard hair were harvested using a needle that allowed convenient injections of FUs to reconstruct eyebrows since beard hair grafts contain 1 hair per unit [12]. However, the characteristics of beard hair (such as caliber and growth rate) are more similar to those of occipital hair than to hair from other parts of the body, making beard hair more suitable for scalp hair restoration. Consequently, beard hair may now be used to treat androgenetic alopecia (AGA) and scar tissues, with a reported survival rate of nearly $80 \%[9,10,13,14]$. It is possible that the beard could supply as many as 9000 grafts [10]. However, the surgical outcomes of using beard hair have been variable, depending on complications encountered at the donor site.

Patients with different ethnic backgrounds have different skin and hair biology characteristics, such as irritation reactions and wound healing $[15,16]$. In this context, a range of complications have been observed in different populations following beard hair extraction. In a study of Caucasian patients, Umar [7] reported no complications in 122 cases of body hair transplantation, including no visible hypopigmentation or keloid scarring. In a study of Indian patients, Saxena and Savant [6] observed hypopigmented scars and folliculitis in the donor area below the jawline of those patients with darker skin, although far fewer beard hairs were extracted than reported in earlier studies. In another study, Indian researchers restricted the safe donor site for the beard area to the submandibular area even though beard hairs are also distributed in the cheek and neck area with a density of $49.7 \mathrm{FUs} / \mathrm{cm}^{2}$ [11]. Additionally, given the common habit of shaving the beard area, when a large number of beard hairs are extracted, the occurrence of hypopigmentation in the beard site is more visible than in the 
occipital site, which could be covered by the remaining hair.

Recently, beard hair has shown tremendous potential in the field of hair transplantation. However, it has not been studied extensively in East Asian men. Therefore, we designed the present study to investigate the efficacy and safety of large-scale beard hair extraction for scalp hair restoration in East Asian men using retrospective patient data compiled in our department. The results of the study confirm that large-scale beard hair extraction acts as another safe and effective donor site to support scalp hair restoration in East Asian males.

\section{METHODS}

\section{Subjects}

Data on male patients with severe alopecia and a thick beard who were treated by the Department of Plastic and Aesthetic Surgery (Nanfang Hospital of Southern Medical University) were collected for the period between March 2017 and December 2018. The area of balding and the occipital FU density were evaluated preoperatively. The distribution of beard hair and the FU density were also assessed. All patients presented no history of hypertension, heart disease, diabetes, or cicatricial diathesis.

The study was approved by the Institutional Review Board of Nanfang Hospital, and the study was conducted in accordance with the principles of the Declaration of Helsinki of 1964 and its later amendments. All participants gave informed consent before study participation. The patients also signed a consent form providing permission to include clinical photographs in this article.

\section{Surgical Technique}

Patients were asked to avoid shaving their beard for at least 1 to 2 days prior to the transplantation procedure. At the time of the procedure, hairs from the donor areas of the occipital and beard areas were trimmed to a length of $1 \mathrm{~mm}$ and disinfected with chlorhexidine lotion.
Tumescent fluid containing $0.2 \%$ lidocaine and $1: 100,000$ epinephrine was then injected into the subcutaneous layer of the donor site, with or without $2 \%$ lidocaine as a local anesthetic. Specifically, for the beard site, local anesthesia was placed along the border of the planned donor area before the tumescent fluid was injected. FU grafts were obtained from the donor sites using a hollow punch (outer diameter of $0.8-0.9 \mathrm{~mm}$ for beard hair and $1 \mathrm{~mm}$ for scalp hair) that was mechanically rotated utilizing a motorized system (Fig. 1). Beard FUs were harvested from the cheek, mandibular area, submandibular area, and anterior neck (Fig. 2). The direction, angle, and depth of the punch were adjusted based on the orientation of the residual hairs. Each freed FU was pulled out using forceps and placed on a gauze pad in a petri dish that was soaked with chilled Ringer's solution. The recipient site was tumefied before slits were made using a 20G needle. A mixture of occipital and beard FUs were implanted in the designated areas and distributed accordingly to a planned density of $35-45 \mathrm{FUs} / \mathrm{cm}^{2}$. In particular, the hairline area was populated only with single-hair occipital FUs; mixed occipital and beard FUs were transplanted in the

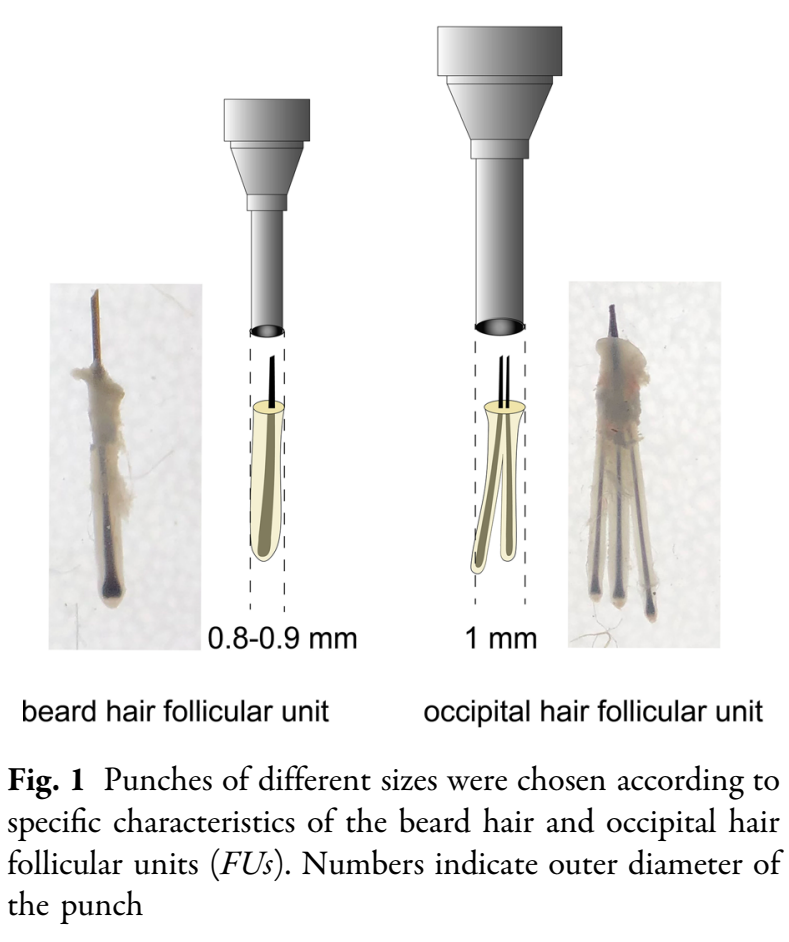




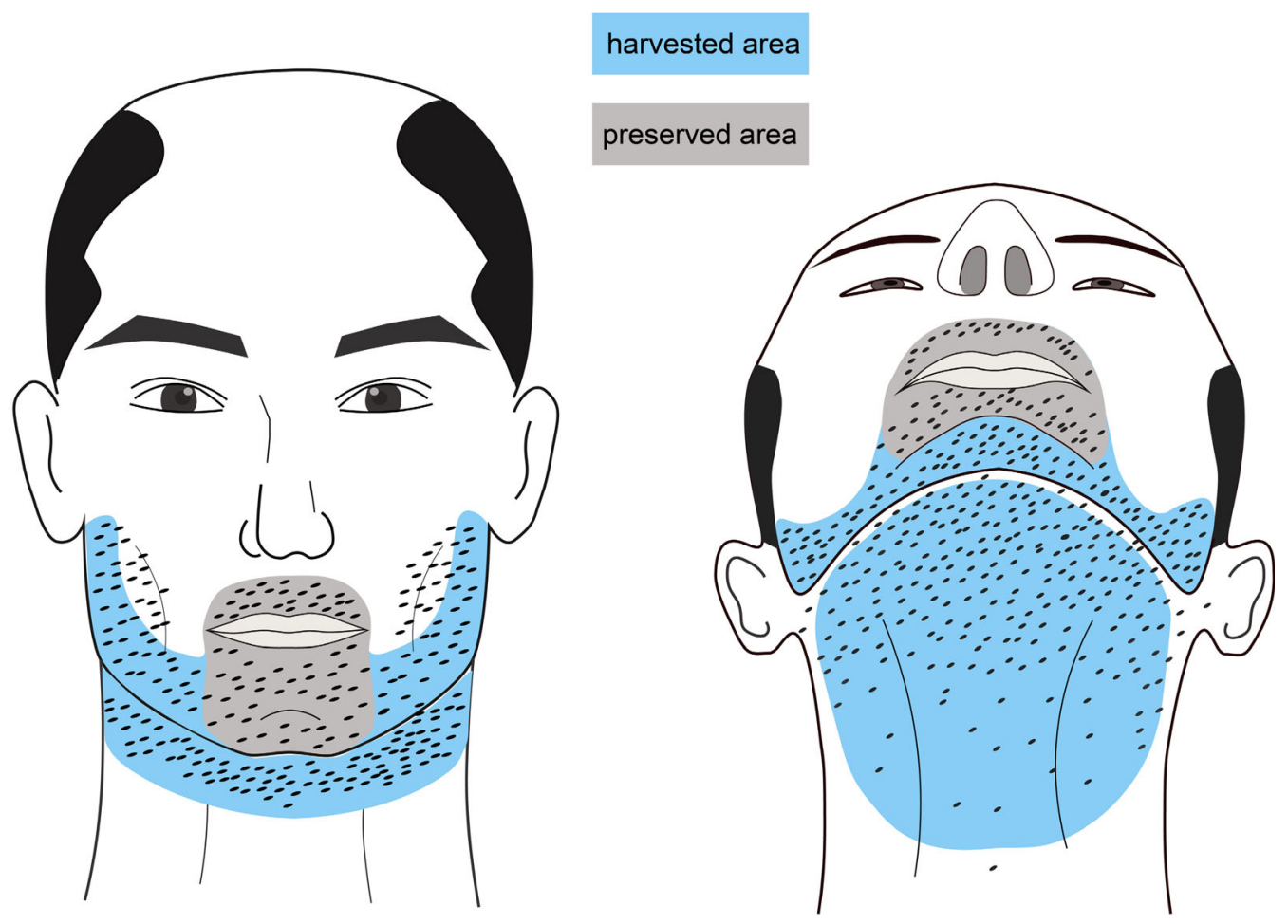

Fig. 2 The most commonly harvested beard areas included the cheek, mandibular, submandibular, and anterior neck areas (blue shading). The perioral area (gray shading) should be avoided during beard hair harvesting

remaining balding zone (Fig. 3). The beard area used as the donor site was tattooed to identify the location to evaluate future beard hair regrowth. Topical antibiotics were applied to

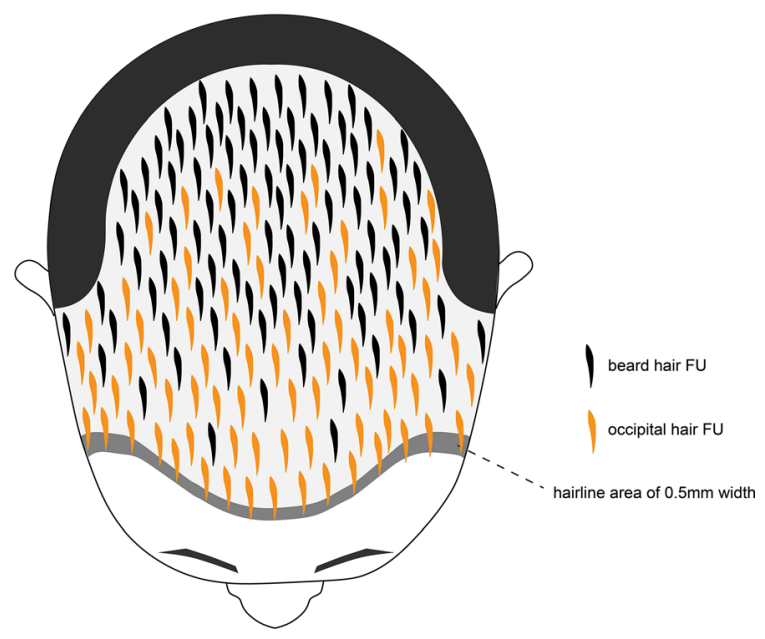

Fig. 3 Only one-hair occipital FUs could be transplanted to the hairline area. Both beard and occipital FUs could be distributed to the remaining bald area the donor site for 2-3 days until the dressing was removed on the third postoperative day. The patients were counseled not to wash their transplanted hair until 2-3 days after the procedure.

\section{Follow-Up}

At 3-5 days and 1 month following the procedure, each donor and recipient site was assessed for the occurrence of any complications, which were recorded as none, folliculitis, or infection. At 9 months postoperatively, the survival rate of all transplanted FUs was evaluated, as was the visibility of the scars of the punch wounds in the beard area. Patient satisfaction was documented at the same time.

\section{Data Collection}

Specific characteristics of the patients that were recorded included: total number of subjects; age of subject; Norwood-Hamilton classification; 
and cause of alopecia. The FU density of the donor site was recorded prior to surgery. During the surgery, the number of grafts and the duration of the procedure were recorded. Specific data on harvesting time, transection rate, and graft yield from the occipital and beard areas were collected separately. Data on complications, survival rates of transplanted FUs, and patient satisfaction were obtained at the corresponding follow-up examinations, in addition to a record of the visibility of the hypopigmented scar.

\section{Statistical Analysis}

Quantifiable data were described as the mean \pm standard deviation and enumerate data were described as rate. Univariate analysis was conducted using the Chi-square test. A $P$ of $<0.05$ was considered to be statistically significant.

\section{RESULTS}

A total of 36 male Chinese patients were included in this study (Table 1). All subjects were diagnosed with AGA, with a Norwood-Hamilton scale classification of male pattern baldness of V-VI [17]. Mean age was 37 (range 3545 ) years (Table 1 ).

Each surgery was carried out by a team composed of one or two surgeons with three to four assistants. Significant differences in the mean FU density were observed between the

Table 1 Patient characteristics

\begin{tabular}{ll}
\hline Patient characteristics & Values \\
\hline Ethnicity (Chinese/others), $n$ & $36 / 0$ \\
Age (years) & $37 \pm 5$ \\
Norwood-Hamilton classification (V/VI), $n$ & $14 / 22$ \\
Total number of subjects in study & 36 \\
Causes of alopecia (AGA/others), $n$ & $36 / 0$ \\
\hline
\end{tabular}

Values are presented at the mean \pm standard deviation (SD) unless indicated otherwise

$A G A$ Androgenetic alopecia occipital and beard areas $(78.6 \pm 4.6$ vs. $48.4 \pm 9.3 \mathrm{FU} / \mathrm{cm}^{2}$, respectively). Consequently, fewer FUs were harvested from the occipital sites than from the beard sites $(3135 \pm 863$ vs. $2352 \pm 599$ FUs $)$; these FUs took approximately $3.1 \pm 0.9$ and $2.1 \pm 0.6 \mathrm{~h}$, respectively, to collect. The beard grafts were obtained from the submandibular and anterior neck areas (Table 2). The mean number of FUs of the total transplanted hair grafts was $5487 \pm 812$; these FUs were collected in a single procedure. The rate of transection for occipital FUs was $3.7 \pm 0.4 \%$, which was significantly less than the transection rate of $3.9 \pm 0.2 \%$ for beard FUs. In most cases, the planned recipient density was set at $35-45 \mathrm{FUs} / \mathrm{cm}^{2}$. The mean time needed for the entire procedure of collecting and transplanting $5487 \pm 812$ FUs was $10.0 \pm 0.9 \mathrm{~h}$ (Table 3 ).

The transplanted grafts regrew well at the recipient sites. Compared to the 3-day postoperative results, dermoscopic observation at 1 month postoperatively revealed that both occipital and beard grafts were lost to some degree (Fig. 4a-h). However, at 9 months postoperatively, healthy and dense FU grafts were observed to cover the entire recipient site (Fig. $4 \mathrm{a}-\mathrm{h}$ ). The beard hairs were observed to be thicker than the occipital hairs (Fig. 4a-h). Therefore, with the sole distribution of one-hair occipital grafts at the hairline area, a natural, visual appearance was achieved. The mixed-hair distribution in the vertex area contributed to the thick appearance of the hair (Fig. 4i-k). The survival rate for the mixed grafts was $95.7 \pm 1.6 \%$, which correlated with the visual appearance.

The donor sites of both the occipital and beard areas healed well. Specifically, the postoperative area was covered sufficiently by the residual hairs. The hair in the donor sites appeared thinner, but no alopecia was observed. Little to no hypopigmentation was noted in any of the patients (Table 4; Fig. 5).

No infection was observed at either the recipient or donor sites 3-5 days following surgery. Two and four patients experienced mild folliculitis in the occipital and beard areas, respectively; no complaints were recorded from the remaining patients (Table 5). All patients 
Table 2 Characteristics of surgery according to harvested area

\begin{tabular}{lccc}
\hline Chararacteristics & Occipital area & Beard area & $\boldsymbol{P}$ value \\
\hline FU density $\left(\mathrm{FU} / \mathrm{cm}^{2}\right)$ & $78.6 \pm 4.6$ & $48.4 \pm 9.3$ & $<0.05$ \\
Graft amount $(\mathrm{FU})$ & $3135 \pm 863$ & $2352 \pm 599$ & $<0.05$ \\
Harvesting time $(\mathrm{h})$ & $3.1 \pm 0.9$ & $2.1 \pm 0.6$ & $<0.05$ \\
Transection rate $(\%)$ & $3.7 \pm 0.4$ & $3.9 \pm 0.2$ & $<0.05$ \\
\hline
\end{tabular}

Values in table are presented as the mean $\pm \mathrm{SD}$

Table 3 Characteristics of surgery-transplantation

\begin{tabular}{ll}
\hline Characteristics & Values \\
\hline Graft amount $(\mathrm{FU})$ & $5487 \pm 812$ \\
Recipient density $\left(\mathrm{FU} / \mathrm{cm}^{2}\right)$, range & $35-45$ \\
Length of operation $(\mathrm{h})$ & $10.0 \pm 0.9$ \\
\hline
\end{tabular}

Values in table are presented as the mean \pm SD unless stated otherwise

were either satisfied or very satisfied with the cosmetic results at 9 months after the surgery (Table 6).

\section{DISCUSSION}

We have examined a possible solution for a deficit of donor grafts in East Asian patients with advanced AGA and investigated the safety and efficacy of large-scale beard hair extraction in East Asian men with advanced AGA. Our results show that large-scale beard hair extraction among our East Asian male subjects was safe and that it left little to no visible hypopigmented scars in the beard area. Moreover, the extracted beard FUs enhanced the cosmetic appearance in the balding area when combined with occipital follicular grafts and also avoided overextraction of FUs in the occipital area. We observed that it was important to avoid transplanting beard FUs to the hairline area because the thickness of beard hair could result in an unnatural appearance at the hairline.

The potential yields of FUs for grafting from the beard area are quite variable and dependent on the number of FUs required. Some investigators have recommended not collecting a large number of FUs from the beard area in patients with darker pigmented skin due to the possibility that visible hypopigmented scars could develop [6, 7]. In another study, a small number of beard FUs were transplanted to strip scars in the scalps of Indian patients, but the results were not entirely satisfactory due to the different characteristics of beard hairs and scalp hairs [9]. A reliable beard site was evaluated by Indian researchers and found to provide approximately 824 FUs [11]; in comparision, in Caucasians, a larger number of FUs can be collected from a beard site. Umar [10] harvested 9000 beard hairs in a hirsute patient who had experienced several failed transplantations and observed no visible hypopigmentation. In another study the FU survival rate reached $80 \%$ when combined with other grafts [8]. Thus, for patients whose occipital grafts are inadequate, beard hairs could be an important source for FUs used in transplantation. In our study, we harvested a mean of $3135 \pm 863$ occipital FUs and $2352 \pm 599$ beard FUs, which were transplanted as mixtures to treat advanced AGA (NorwoodHamilton V-VI) in East Asian patients. This large number of harvested beard FU grafts has not been previously reported in East Asian men. All patients included in this study were satisfied with their final results. The additional beard FU grafts increased the total number of grafts that were available, thereby enhancing hair thickness in the recipient sites. Importantly, no visible hypopigmentation was observed during the postoperative period in any of the subjects in this study. These results suggest that large-scale beard FU collection is safe in East Asian men 

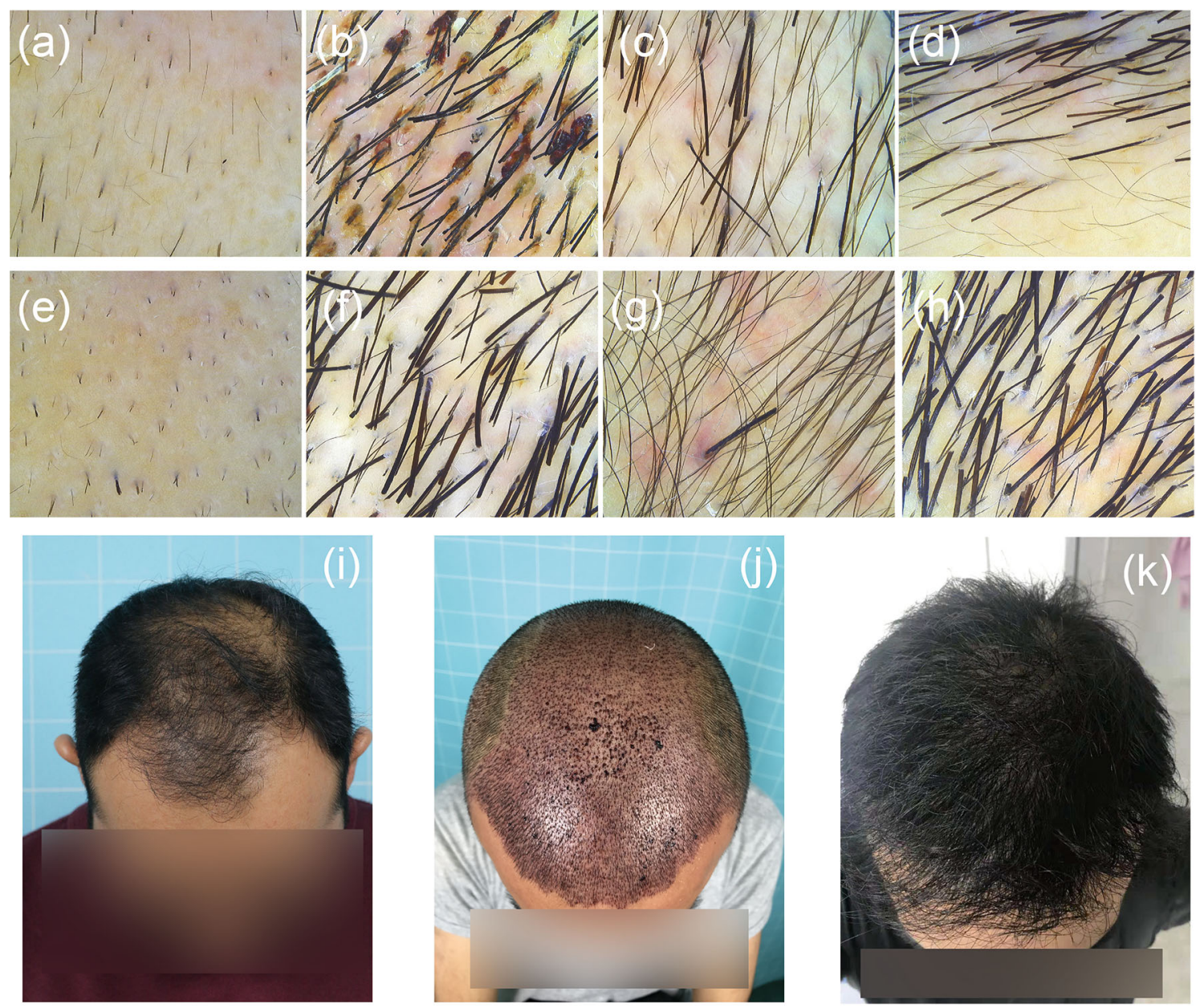

Fig. 4 Regrowth of transplanted occipital and beard grafts. a-h Dermoscopic photographs of transplanted one-hair occipital FUs at the hairline area $(\mathbf{a}-\mathbf{d})$ and beard FUs at the vertex area $(\mathbf{e}-\mathbf{h}) . \mathbf{i}-\mathbf{k}$ Photographs of 45 -year-old male diagnosed with androgenetic alopecia (AGA)

Table 4 Visibility of hypopigmented scars

\begin{tabular}{lc}
\hline Visibility of hypopigmented scars & Number of subjects \\
\hline None & 30 \\
Hardly visible & 6 \\
Less visible & 0 \\
Visible & 0 \\
Total & 36 \\
\hline
\end{tabular}

(Norwood-Hamilton VI classification) who underwent hair transplantation with 3656 occipital FUs and 2765 beard FUs: recipient site before transplantation (i), 3 days after transplantation (j), and 9 months after transplantation $(\mathbf{k})$

and that it effectively enhances hair transplantation.

To our knowledge, the use of only beard hairs for transplantation in scalp hair restoration procedures has been reported only rarely in the published literature. Given the differences in color and other physical characteristics between beard and scalp hair-although the differences are fewer than between beard hair and body hair from other parts of the bodybeard hairs should not be transplanted at too high a density $[6,9]$. In other trials, beard hairs were mixed with other body hairs for transplantation, but the survival rate for such mixed 


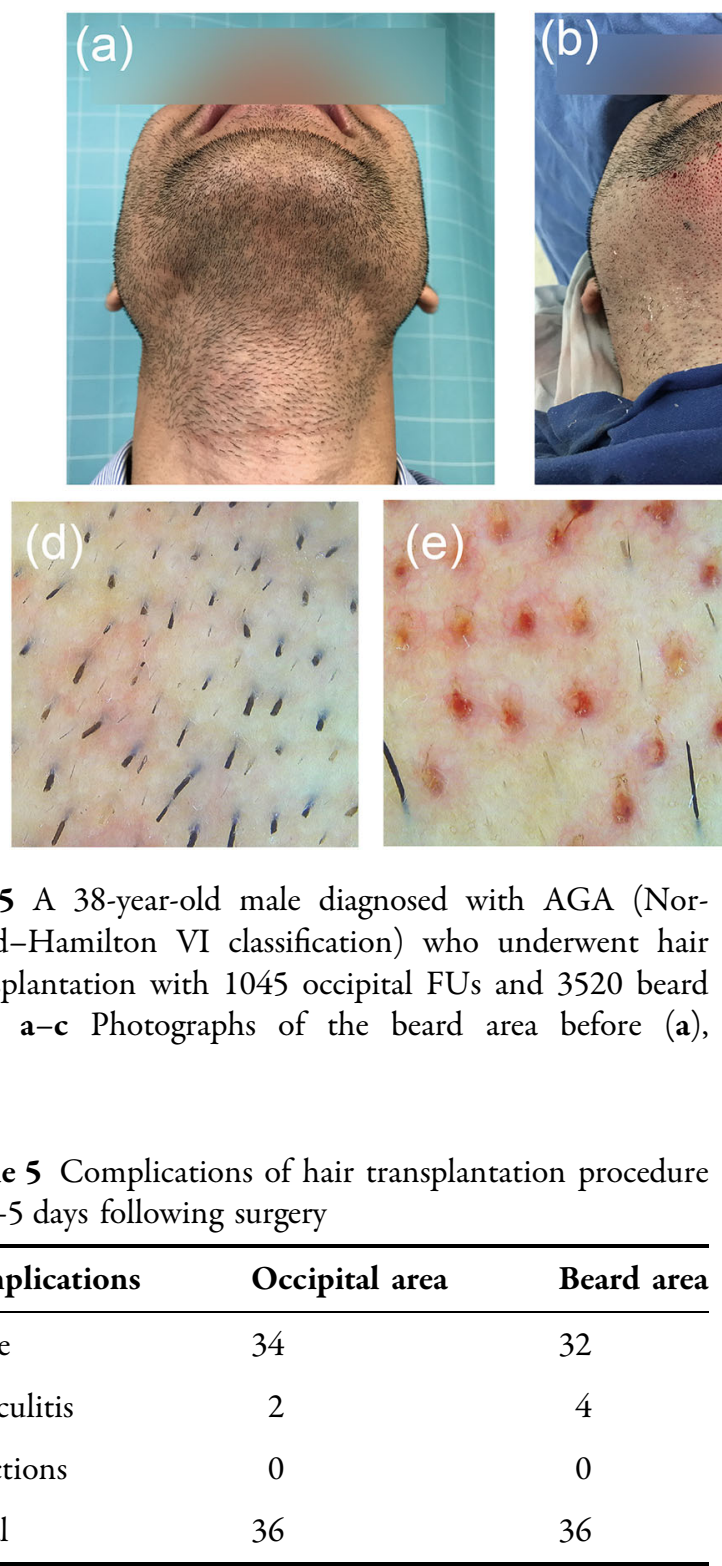

Values are presented as the number of subjects

grafts was approximately $80 \%$ [10]. This result may be associated with the short anagen phase of other body hair, which lasts about 16 weeks, while beard and scalp hair continue to elongate for years [6]. It should be noted that in our study, we obtained a $95.7 \pm 1.6 \%$ survival rate using a combination of only beard and occipital FUs for grafting. With this specific combination, the survival rate of transplanted grafts could be improved, the number of FUs available for grafting could be increased, and overcollection

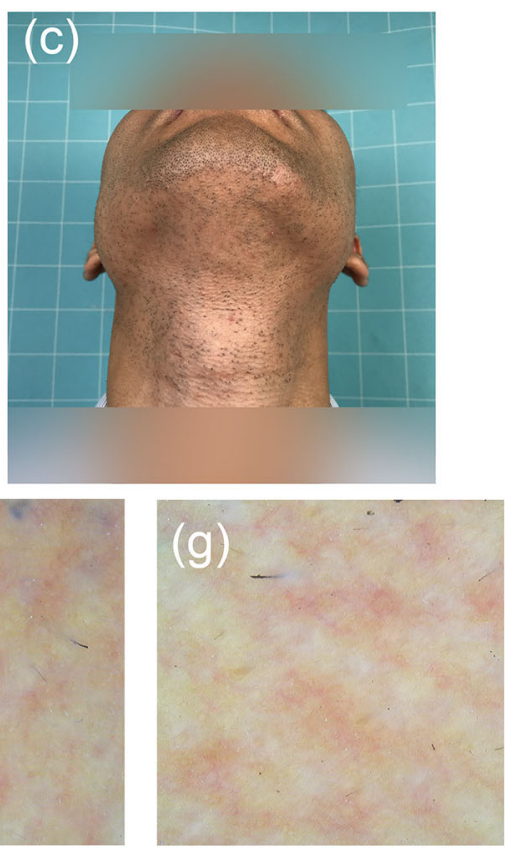

immediately after (b), and 9 months after (c) large-scale beard hair extraction. $\mathbf{d}$-g Dermoscopic photographs of the beard area before (d), 3 days (e), 1 month (f), and 9 months ( $(\mathbf{g})$ after large-scale beard hair extraction

Table 6 Patient satisfaction with the cosmetic results at 9 months after surgery

\begin{tabular}{lc}
\hline Patient satisfaction & Number of patients \\
\hline Very satisfied & 34 \\
Satisfied & 2 \\
Neither satisfied nor dissatisfied & 0 \\
Dissatisfied & 0 \\
Very dissatisfied & 0 \\
Total & 36 \\
\hline
\end{tabular}

of occipital hair could be avoided. Therefore, a mixture of beard and scalp hairs could result in a better outcome for scalp hair restoration.

Beard hairs are distributed on the cheek, mandibular and submandibular areas, and the neck. To obtain large numbers of hair follicles, such as the $2352 \pm 599$ FUs that were used in our study, it is common to use all the abovementioned beard donor sites However, it should be noted that the orientation, angle, and 
pattern of beard hairs vary among these different beard areas. Thus, it is important that the hairs to be collected extend far enough above the skin to enable accurate assessment of these characteristics, as this will decrease the risk of transection during collection.

The size of the harvesting instrument also influenced the transection rate. Both 18- and 19-gauge needles are used more often with Caucasian patients, while 20-gauge needles are used with patients with darker skin, based on the differences in complication rates associated with different skin types. We determined that a hollow punch with an outer diameter of 0.8-0.9 $\mathrm{mm}$ (thinner than a 20-gauge needle) was better suited for East Asian patients and resulted in little to no hypopigmentation in the donor area. In addition, we observed a transection rate of $3.9 \pm 0.2 \%$. The low transection rate also could be attributed to specific characteristics of one-hair follicles or the use of tumescent fluid in the procedure.

The perioperative treatment is also important to achieve successful final results with beard hair transplantation. Minoxidil, one of the two US Food and Drug Administration-approved medications for alopecia, can be used to enhance beard hairs that are available for transplantation $[13,18]$. As a pretreatment before collecting beard FUs, some surgeons apply minoxidil to the beard area for periods ranging from 6 weeks to 6 months $[10,18]$. To specifically target the anagen beard, preshaving could be scheduled 7-10 days prior to surgery $[10,18]$, and the harvesting of beard hairs could be performed under local anesthesia without the need for tumescence. However, we observed that the tight skin that resulted from the use of the tumescent fluid was better for punching, helped decrease the transection rate, and likely reduced the total amount of time needed to complete the procedure. In the postoperative period, the use of laser treatments 2 weeks after transplantation, in addition to antibiotic ointment, may accelerate wound healing in the beard area [10].

In some cultures, the presence of a beard enhances the perception of masculinity in men [19]. In some regions, such as Saudi Arabia [20], most adult males have a mustache and would prefer it to as thick as possible [21]. Moreover, men in some professions, such as academia and the arts, may feel that their strength and selfconfidence would be improved if they had a thick beard. Therefore, it is necessary to retain sufficient beard hair after extraction to not significantly diminish the appearance of the beard and also to avoid the development of hypopigmentation. Beard hair transplantation is not common in Chinese society at the present time. It should also be noted that the distribution and density of beard hair in adult males of China and other East Asian countries are much less compared with adult men of other races [22]. It also is common for East Asian men to shave their beards for personal hygiene [23]. Therefore, the possible use of beard hair for transplantation is sometimes underestimated. According to our study, it is clear that beard FUs are a very effective and safe source for hair grafts in East Asian men with advanced alopecia, and we suggest that the use of beard hair for transplantation deserves more attention.

\section{Limitations}

There are a number of limitations to our study. The number of subjects for whom we had data was small, and only Chinese patients were included. More clinical, well-controlled studies are needed to investigate additional details in the future, such as possible differences in FUs within the beard area and the threshold of beard graft yield. Additional optimization of the procedures that were used could produce better results. Furthermore, it would be useful to follow the patients for a longer period of time to better assess the long-term effects.

\section{CONCLUSION}

In summary, the results of this study confirm that large-scale beard extraction is a safe and effective treatment to enhance the cosmetic result of traditional hair transplantation in East Asian men with advanced alopecia. However, due to the small number of subjects included in this study, additional, randomized, well-controlled studies that focus on specific details of 
the procedure may be required to optimize the outcome.

\section{ACKNOWLEDGEMENTS}

The authors would like to thank the participants of this study.

Funding. This study was funded by Natural Science Foundation of China (Grant No. 81772104, No. 81701929) and the Natural Science Foundation of Guangdong Province (Grant No. 2015A030311001, No. 2017A030310120). The Rapid Service Fee was funded by Natural Science Foundation of China (Grant No. 81772104, No. 81701929) and the Natural Science Foundation of Guangdong Province (Grant No. 2015A030311001, No. 2017A030310120).

Medical Writing, Editorial, and Other Assistance. Writing and editorial assistance in the preparation of this manuscript were provided by EditSprings (Wuhan, China). Support for this assistance was funded by the Natural Science Foundation of China (Grant No. 81772104, No. 81701929).

Authorship. All named authors meet the International Committee of Medical Journal Editors (ICMJE) criteria for authorship for this article, take responsibility for the integrity of the work as a whole, and have given their approval for this version to be published. All authors have full access to all of the data in this study and take complete responsibility for the integrity of the data and accuracy of the data analysis.

Disclosures. De-Cong Zhu, Ye He, ZheXiang Fan, Jin Wang, Qian Qu, Zhi-Qi Hu and Yong Miao have nothing to disclose.

Compliance with Ethics Guidelines. The study was approved by the Institutional Review Board of Nanfang Hospital and the study was conducted in accordance with the principles of the Declaration of Helsinki of 1964 and its later amendments. All participants gave informed consent before study participation. The patients also signed a consent form providing permission to include clinical photographs in this article.

Data Availability. The data generated during and/or analyzed during the present study are available on reasonable request.

Open Access. This article is distributed under the terms of the Creative Commons Attribution-NonCommercial 4.0 International License (http://creativecommons.org/licenses/ by-nc/4.0/), which permits any noncommercial use, distribution, and reproduction in any medium, provided you give appropriate credit to the original author(s) and the source, provide a link to the Creative Commons license, and indicate if changes were made.

\section{REFERENCES}

1. Unger WP, Unger RH. Hair transplanting: an important but often forgotten treatment for female pattern hair loss. J Am Acad Dermatol. 2003;49: 853-60.

2. Stough DR. Hair transplantation. JAMA. 1969;208: 154.

3. Nakatsui T, Wong J, Groot D. Survival of densely packed follicular unit grafts using the lateral slit technique. Dermatol Surg. 2008;34(1016-22): 1022-5.

4. Lee W, Lee S, Na G, et al. Survival rate according to grafted density of Korean one-hair follicular units with a hair transplant implanter: experience with four patients. Dermatol Surg. 2006;32:815-8.

5. Harris JA. Follicular unit extraction. Facial Plast Surg Clin North Am. 2013;21:375-84.

6. Saxena K, Savant SS. Body to scalp: evolving trends in body hair transplantation. Indian Dermatol Online J. 2017;8:167-75.

7. Umar S. Body hair transplant by follicular unit extraction: my experience with 122 patients. Aesthet Surg J. 2016;36:1101-10.

8. Meyer-Gonzalez T, Bisanga C. Body-hair transplant for cicatricial alopecia. Actas Dermosifiliogr. 2012;103:163-4. 
9. Poswal A. Use of body and beard donor hair in surgical treatment of androgenic alopecia. Indian J Plast Surg. 2013;46:117-20.

10. Umar S. Hair transplantation in patients with inadequate head donor supply using nonhead hair: report of 3 cases. Ann Plast Surg. 2011;67:332-5.

11. Chouhan K, Kota RS, Kumar A, Gupta J. Assessment of safe donor zone of scalp and beard for follicular unit extraction in Indian Men: a study of 580 cases. J Cutan Aesthet Surg. 2019;12:31-5.

12. Hirai $\mathrm{T}$, Inoue $\mathrm{N}$, Nagamoto $\mathrm{K}$. Potential use of beards for single-follicle micrografts: convenient follicle-harvesting technique using an injection needle. Ann Plast Surg. 2001;47:37-40.

13. Umar S. Use of beard hair as a donor source to camouflage the linear scars of follicular unit hair transplant. J Plast Reconstr Aesthet Surg. 2012;65: 1279-80.

14. Akdag O, Evin N, Karamese M, Tosun Z. Camouflaging cleft lip scar using follicular unit extraction hair transplantation combined with autologous fat grafting. Plast Reconstr Surg. 2018;141:148-51.

15. Roberts WE. Skin type classification systems old and new. Dermatol Clin. 2009;27:529-33.

16. Lancer HA. Lancer ethnicity scale (LES). Lasers Surg Med. 1998;22:9.
17. Norwood OT. Male pattern baldness: classification and incidence. South Med J. 1975;68:1359-65.

18. Ingprasert $\mathrm{S}$, Tanglertsampan $\mathrm{C}$, Tangphianphan $\mathrm{N}$, Reanmanee C. Efficacy and safety of minoxidil 3\% lotion for beard enhancement: a randomized, double-masked, placebo-controlled study. J Dermatol. 2016;43:968-9.

19. Dixson BJ, Rantala MJ. The role of facial and body hair distribution in women's judgments of men's sexual attractiveness. Arch Sex Behav. 2016;45: 877-89.

20. Hsu TS, Davis MD, El-Azhary R, Corbett JF, Gibson LE. Beard dermatitis due to para-phenylenediamine use in Arabic men. J Am Acad Dermatol. 2001;44: 867-9.

21. Straub PM. Replacing facial hair. Facial Plast Surg. 2008;24:446-52.

22. Wu Y, Konduru R, Deng D. Skin characteristics of Chinese men and their beard removal habits. Br J Dermatol. 2012;166 Suppl 1:17-21.

23. Maurer M, Rietzler M, Burghardt R, Siebenhaar F. The male beard hair and facial skin-challenges for shaving. Int J Cosmet Sci. 2016;38 Suppl 1:3-9. 